\title{
Neuropathic Pain in A Patient with Relapsing-Remitting Multiple Sclerosis - A Case Report
}

\author{
Goce Kalcev $^{1 *}$, Ivan Barbov ${ }^{1}$ and Elizabeta Zisovska ${ }^{2}$ \\ ${ }^{1}$ University Clinic for Neurology, Skopje, Republic of North Macedonia \\ ${ }^{2}$ University Clinic for Gynecology and Obstetrics, Skopje, Republic of North Macedonia \\ *Corresponding Author: Goce Kalcev, University Clinic for Neurology, Skopje, \\ Republic of North Macedonia.
}

Received: February 14, 2020

Published: March 06, 2020

(C) All rights are reserved by Goce Kalcev., et al.

\begin{abstract}
Multiple sclerosis (MS) is an autoimmune disorder characterized by immune cell infiltration and inflammation within the central nervous system (CNS) causing progressive demyelination and neurodegeneration. Neuropathic pain in patients with MS is of central origin. It is well known that neuropathic pain has a negative impact on MS patients' quality of life. Therefore, we present a case of a 40- year-old man from Republic of North Macedonia with Relapsing-remitting MS (RRMS). The patient was asked to evaluate how the neuropathic pain influenced the enjoyment of life, daily activity, walking, mood, sleep, work and relations with others. This type of pain mostly interfered with patient enjoyment of life. Clearly, neuropathic pain generally disturbs the comfort of patients with multiple sclerosis.
\end{abstract}

Keywords: Multiple Sclerosis; Neuropathic Pain; Evaluation; Demyelination; Neurodegeneration

\section{Introduction}

Multiple sclerosis (MS) is an autoimmune disorder characterized by immune cell infiltration and inflammation within the central nervous system (CNS). Relapsing-remitting MS and secondary progressive MS cause progressive demyelination and neurodegeneration [1]. Today we know the autoimmune origin of the disease, especially the involvement of auto-aggressive $\mathrm{T}$ cells directed against neuronal myelin [2]. Women are affected twice as often as men. The mean age of MS onset is 30 years of age; women generally fall ill earlier than men [3]. It is characterized by a large spectrum of symptoms and signs, involving several functional systems (pyramidal, cerebellar, sensory, brainstem, bowel and bladder, visual, mental, and ambulation) [4]. It is well known that neuropathic pain has a negative impact on MS patients' quality of life. Neuropathic pain is most directly related to the pathology of MS and its prevalence has been estimated to be $29 \%$ in a meta-analysis [5]. Previous studies on the prevalence of neuropathic pain in the early stages of MS found a point prevalence of $14 \%$ and a lifetime prevalence of $28 \%$ [6].
Materials and methods: Processing of a patient who was admitted to the University Neurology Clinic with neuropathic pain, previously diagnosed with relapsing-remitting MS (RRMS).

\section{Case Report}

Two years ago, 40 years old, the male patient appeared at the Neurology Clinic in Skopje, due to intermittent tingling on the left half of the face and left hand, lasting 1 to 2 minutes. They were repetitive several times a day, sometimes up to 20 times. While he was in the army (15 years ago) he was ordered to clean the toilets, due to the powerful chemicals they used, he had tingling and weakness on the left side of the body, feeling of burning and electricallike sensations. Also, the patient reported problems of the type "not looking at the sides" and blurry visions. During the hospitalization, the patient was treated with pulse doses of corticosteroids. A year later, the patient was hospitalized for the second time due to the tingling of the right side of the body, tremor of the right leg and difficulties in walking. After the diagnostic tests performed (MR 
and KT), he was diagnosed with multiple sclerosis. Since then, he has been receiving Immunomodulatory therapy. This therapy has been shown to reduce the progression of MS. He was hospitalized again at the Neurology Clinic in Skopje due to a relapse that manifested itself with an appearance of tingling on both legs, accompanied by an occasional spasm in the right limb and right knee. The symptoms appeared at first on the fingers of the feet, which after a few days spread to the knees. The patient felt a spasm in the right knee the day before hospitalization. Laboratory investigations showed the following pathological findings: Interferon antibody-beta (5.018.40 pg/ml); Glucose (8.9 mmol/l); Cholesterol (5.3 mmol/l); Vitamin D3 (63, $90 \mathrm{ng} / \mathrm{ml})$. Visual evoked potentials showed responses bilaterally with prolonged latencies. The somatosensory evoked potentials showed that the cortical response is morphologically altered with a boundary latency that is in favor of a conduction defect across the central paths to the right. Paresthesia of both legs appeared to the height of the knee. Due to the high titer of interferon antibodies and relapses in the year after year, it was required a change with drugs from the second line. Magnetic resonance imaging of the brain, a natural and post-contraction series has been made. Bilateral periventricular and subcortical fibers showed multiple hyper signal changes in T2 and FLER, which according to their distribution were suitable for demyelination. Changes were located periventricular to the left occipital horn of the latent chamber, as well as in the projection of the posterior horn of the inner and right cerebral pelvic cavity capsule showed a restriction of diffusion. A similar change was also made in the projection of the insula left. Computer tomography showed the following pathological findings: On the level L4 - L5, a light dorsomedial infiltration of the intervertebral disk is noted. Mild dorsomedial protrusion of the intervertebral disk (grade 2) is noticed at level L5-C1.

\section{Discussion}

It is well known that throughout the evolution of the disease, patients diagnosed with multiple sclerosis can have an important physical disability, but also neuropsychological symptoms such as depression and anxiety impaired quality of life [7]. The overall prevalence of pain in patients diagnosed with MS is between $40 \%-65 \%$. This is considered to be an important factor that has an impact on the overall health-related quality of life of MS patients $[8,9]$. Factors which are considered to contribute to pain severity in patients diagnosed with MS are: gender, age, educational level, a grade of disability and increased health care use [10]. In addition to this, the patient was asked to evaluate how the neuropathic pain influenced enjoyment of life, daily activity, walking, mood, sleep, work and relations with others. These items were also assessed using a numeric scale. The patient was instructed to grade each item by " 0 " if the pain did not interfere with that specific item and by " 10 " if it interfered completely with that specific item. The patient suffers most from tingling, cold-like sensations, electrical-like sensations and tremor. Neuropathic pain mostly interfered with patient enjoyment of life. The most common sites of neuropathic pain were lower extremities, followed by upper extremities. The maximum pain duration was 6 days and a minimum of 2 days per month. Neuropathic pain can be defined as a process that happens after a primary lesion or as a disease of the somatosensory nervous system [10]. Neuropathic pain is usually chronic, either persisting continuously or characterized by recurrent painful episodes [11]. Central neuropathic pain is caused by a lesion or disease of the spinal cord and/or brain [10]. Following the protocol (evidencebased medicine) anticonvulsant and antidepressants are used to treat neuropathic pain. For the treatment and reducing the neuropathic pain, the patient takes anticonvulsant drugs (gabapentin, $1800 \mathrm{mg}$ per day) and antidepressants or tried to rest in order to reduce pain. Even though neuropathic pain is a common symptom in patients diagnosed with MS, in most of the cases, it still does not get the proper attention and consideration. The patient also complained of pain in the eyes, reduced vision, not looking at the side at all. This was the occasion for the patient to be examined by an ophthalmologist. The ophthalmologist concluded that there was an early phase of peri phlebitis with hemorrhages, areas of ischemia, and neovascularization.

\section{Conclusion}

With the description of our patient above, we hope we have captured how much the neuropathic pain disturbs the comfort of patients with multiple sclerosis. As shown above, the pain has a major impact on the quality of life of these patients. This is the reason to do more research with a stronger focus on neuropathic pain, in order to provide a better quality of life for these patients.

\section{Conflict of Interest}

There is not a conflict of interest. Permission to publish this case report was obtained from the patient.

\section{Founding}

None. 


\section{Bibliography}

1. McKinney EF., et al. "Tcell exhaustion, co-stimulation and clinical outcome in autoimmunity and infection". Nature 523 (2015): 612-622.

2. Dendrou CA., et al. "Immunopathology of multiple sclerosis". Nature Reviews Immunology 15 (2015): 545-558.

3. Jakob E., et al. "Uveitis subtypes in a German interdisciplinary uveitis center-analysis of 1916 patients". The Journal of Rheumatology 36.1 (2009):127-136.

4. Goldenberg MM. "Multiple sclerosis review". PT 37 (2012): 175-184.

5. Foley PL., et al. "Prevalence and natural history of pain in adults with multiple sclerosis: systematic review and metaanalysis". Pain 154 (2013): 632-642.

6. Martinelli Boneschi F., et al. "Lifetime and actual prevalence of pain and headache in multiple sclerosis". Multiple Sclerosis Journal 14 (2008): 514-521.

7. CH Marck., et al. "Physical activity and associated levels of disability and quality of life in people with multiple sclerosis: a large international survey". BMC Neurology 14.1 (2014).

8. A Rintala., et al. "Ten-year follow-up of health-related quality of life among ambulatory persons with multiple sclerosis at baseline". Quality of Life Research 25.12 (2016): 3119-3127.

9. Sarraf P., et al. "Relationship between Sleep Quality and Quality of Life in Patients with Multiple Sclerosis". International Journal of Preventive Medicine 12 (2014): 1582.

10. Jensen TS., et al. "A new definition of neuropathic pain". Pain 152 (2011): 2204-2205.

11. Scholz J., et al. "The IASP classification of chronic pain for ICD11: Chronic neuropathic pain". Pain 160 (2019): 53-59.

\section{Assets from publication with us}

- Prompt Acknowledgement after receiving the article

- Thorough Double blinded peer review

- Rapid Publication

- Issue of Publication Certificate

- High visibility of your Published work

Website: https://www.actascientific.com/

Submit Article: https://www.actascientific.com/submission.php

Email us: editor@actascientific.com

Contact us: +919182824667

Citation: Goce Kalcev., et al. "Neuropathic Pain in A Patient with Relapsing-Remitting Multiple Sclerosis - A Case Report". Acta Scientific Medical Sciences 4.4 (2020): 25-27. 